\title{
OPTIMIZATION OF MATCHING PURSUIT ENCODER BASED ON ANALYTICAL APPROXIMATION OF MATCHING PURSUIT DISTORTION
}

\author{
Alireza Shoa and Shahram Shirani \\ Dept. of Elec. and Comp. Eng., McMaster University, Hamilton, Canada \\ shoaah@mcmaster.ca, shirani@mcmaster.ca
}

\begin{abstract}
Distortion of matching pursuit is calculated in terms of matching pursuit encoder parameters for uniformly distributed signals and dictionaries. Then, the MP encoder is optimized using the analytically derived approximation for MP distortion. Our simulation results show that this optimized MP encoder exhibits optimum performance for nonuniform signal and dictionary distributions as well.
\end{abstract}

\section{INTRODUCTION}

Many techniques have been proposed to improve the performance of MP encoder by adapting the parameters of the encoder ([2, 3, 4]). However, due to lack of an accurate approximation for MP distortion, these techniques are mostly based on training the encoder to find the optimal parameters. Even when the analytical approach is taken, a very conservative upper bound for the MP distortion is used to find the optimal parameters ([2, 3]). Additionally, this upper bound must be estimated through experiments for different dictionaries. In this paper, we express the distortion of matching pursuit in terms of MP encoder parameters for uniformly distributed signals and dictionaries. This distortion is a much less conservative upper bound for MP distortion than the one obtained in $[2,3]$. Moreover, we use our derived analytical relation to minimize MP distortion for a given rate and find the optimal number of MP stages and step numbers of quantizers used to quantize the inner product coefficients. Finally, we show that when the MP encoder is optimized for uniformly distributed signals and dictionaries, it exhibits optimum performance for other signal distributions and dictionaries (This is observed from experimental results).

\section{MATCHING PURSUIT}

Matching pursuit is a greedy algorithm that decomposes a signal $f$ into an overcomplete dictionary of bases [1]. At each stage of matching pursuit the dictionary vector $g_{\gamma}$ that results in the maximum inner-product with the residual signal $R^{i} f$ is found. Then, the residual signal is projected on $g_{\gamma}$. This can be written as:

$$
R^{i} f=\left\langle R^{i} f, g_{\gamma}\right\rangle g_{\gamma}+R^{i+1} f
$$

where $R^{i+1} f$ is the new residual signal. At the first stage $R^{0} f$ is replaced by the signal $f$. The matching pursuit iterations continue based on equation (1) until the bit budget is exhausted. Therefore, the signal decomposition can be written as:

$$
f=\sum_{i=0}^{k-1}\left\langle R^{i} f, g_{\gamma_{i}}\right\rangle g_{\gamma_{i}}+R^{k} f
$$

\section{DISTORTION OF MP FOR UNIFORM SIGNALS}

In this section the average distortion of matching pursuit is calculated in terms of MP encoder parameters for uniformly distributed signals and dictionaries.

Let us divide both sides of equation (1) by $\left\|R^{i} f\right\|$ :

$$
\frac{R^{i} f}{\left\|R^{i} f\right\|}=\left\langle\frac{R^{i} f}{\left\|R^{i} f\right\|}, g_{\gamma}\right\rangle g_{\gamma}+\frac{R^{i+1} f}{\left\|R^{i} f\right\|}
$$

and let:

$$
\overrightarrow{r_{i}} \triangleq \frac{R^{i} f}{\left\|R^{i-1} f\right\|} \quad \text { and } \quad r_{i} \triangleq\left\|\overrightarrow{r_{i}}\right\|=\frac{\left\|R^{i} f\right\|}{\left\|R^{i-1} f\right\|}
$$

Equation (3) is the MP equation for the normalized signal $\frac{R^{i} f}{\left\|R^{i} f\right\|}$ and therefore, $r_{i}$ is the norm of the resulting residual signal when matching pursuit is applied to the normalized signal. Substituting $\left\|R^{0} f\right\|$ by $\|f\|$ and using equation (4) repeatedly, we can write:

$$
\left\|R^{k} f\right\|=\|f\| r_{1} r_{2} \ldots r_{k}
$$

The norm of the residual signal at the $k^{\text {th }}$ stage is equal to the total distortion of $k$-stage matching pursuit. Therfore:

$$
D_{\mathrm{MP}}=\left\|R^{k} f\right\|^{2}=\|f\|^{2}\left(r_{1} r_{2} \ldots r_{k}\right)^{2}
$$

In order to find the distortion of matching pursuit, the behavior of the residual norm $r_{i}$ must be studied. In what follows, we first approximate the Voronoi regions in which the vectors $\vec{r}_{i}$ are located. Then, we find the probability distribution of $r_{i}$ and their product $\left(\prod_{i=1}^{k} r_{i}\right)$. Once that probability distribution is available, the expectation of $D_{\mathrm{MP}}$ can be calculated.

Let us consider equation (3) and assume that the dimension of the vector $f$ is $N$. Therefore, $\frac{R^{i} f}{\left\|R^{i} f\right\|}$ is a vector on the surface of a unit $N$-sphere where $N$-sphere is an $N$-dimensional hypersphere. Moreover, since the dictionary elements are normalized $N$ dimensional vectors, they are also on the surface of the unit $N$ sphere. Now suppose the negative of each of the dictionary vectors is included in the dictionary. Therefore, finding the maximum inner-product is equivalent to finding the minimum Euclidean distance. If the size of the original dictionary is $M$, the size of the new dictionary will be $2 M$. Note that this assumption is only for our modeling convenience and is not implemented in practice. In fact, the sign bit of the inner product coefficient is now used to encode the dictionary index for a dictionary of size $2 M$.

Now assume the signal is uniformly distributed on the surface of the $N$-sphere. Since the signal is uniform, for the best performance, the dictionary vectors must be uniformly placed on 


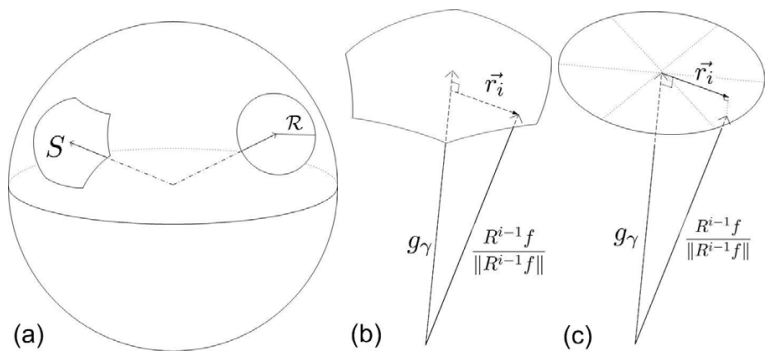

Fig. 1. (a) 3-dimensional Voronoi region is approximated by a two dimensional circle with the same volume as the surface area of the Voronoi region (in two dimensions, Volume $=\pi \mathcal{R}^{2}$, area $=2 \pi \mathcal{R}$ ) (b) Geometric interpretation of the residue $\overrightarrow{r_{i}}$. (c) $\overrightarrow{r_{i}}$ is uniformly distributed in the volume of the 2-dimensional sphere.

the surface of the $N$-sphere. This means that the Voronoi regions for each dictionary vector must be identical and therefore have the same area. Moreover, to maximize uniformity, the Voronoi regions must approach the shape of a spherical cap.

If the total surface area of the unit $N$-sphere is $A_{N}$, since there are $2 M$ Voronoi regions and the Voronoi regions are identical, the surface area of each of the Voronoi regions will be $S=\frac{A_{N}}{2 M}$. For the optimum dictionary these shapes must be as close as possible to the shape of a spherical cap and their surface areas must be as close as possible to $S$. Therefore, when the number of elements in the dictionary is high, it is reasonable to approximate the Voronoi regions by $N-1$ dimensional spheres with the same volume as the surface area of the Voronoi regions $(S)$. This is shown in figure 1.(a). Thus, using the equations for the volume and surface area of hyper-spheres [5], the volume of these $N-1$ dimensional spheres can be written as (the radius of the unit $N$-sphere is 1 ):

$$
V_{N-1}=S=\frac{A_{N}}{2 M} \Rightarrow \frac{\pi^{\frac{N-1}{2}}}{\left(\frac{N-1}{2}\right) !} \mathcal{R}^{N-1}=\frac{N \pi^{\frac{N}{2}}}{\left(\frac{N}{2}\right) ! 2 M}
$$

Therefore, the radius of the $N-1$ dimensional sphere is found by:

$$
\mathcal{R}=\left(\frac{\sqrt{\pi} N\left(\frac{N-1}{2}\right) !}{2\left(\frac{N}{2}\right) !}\right)^{\frac{1}{N-1}} M^{-\frac{1}{N-1}}
$$

Now that the Voronoi regions are modelled for the uniform dictionary, the distribution of the residual norms $\left(r_{i}\right)$ can be found. According to (3) and (4):

$$
\frac{R^{i-1} f}{\left\|R^{i-1} f\right\|}=\left\langle\frac{R^{i-1} f}{\left\|R^{i-1} f\right\|}, g_{\gamma}\right\rangle g_{\gamma}+\overrightarrow{r_{i}}
$$

Therefore, $\overrightarrow{r_{i}}$ is the residual vector for the normalized signal $\frac{R^{i-1} f}{\left\|R^{i-1}\right\|}$. Hence, $\overrightarrow{r_{i}}$ points from the approximation of the normalized signal to a point on the Voronoi region as shown in figure 1.(b). The Voronoi regions are approximated by an $N-1$ dimensional sphere with radius $\mathcal{R}$. Since the input signal is uniformly distributed on the surface area of a unit $N$-sphere, we can assume the residue vector $\overrightarrow{r_{i}}$ is uniformly distributed in the volume of the $(N-1)$-sphere with radius $\mathcal{R}$ as shown in figure 1.(c).

With this assumption, $r_{i}=\left\|\overrightarrow{r_{i}}\right\|$ will be the distance of the point corresponding to vector $\overrightarrow{r_{i}}$ to the center of the sphere. Let $\mathbf{r}_{\mathbf{i}}$ be the random variable corresponding to this distance. We can obtain the probability distribution of $\mathbf{r}_{\mathbf{i}}$ assuming the residual signal is uniformly distributed in the volume of the $(N-1)$-sphere:

$$
F_{\mathbf{r}_{i}}\left(r_{i}\right)=P\left(\mathbf{r}_{i} \leq r\right)=\frac{\frac{\pi^{\frac{N-1}{2}}}{\left(\frac{N-1}{2}\right) !} r_{i}^{N-1}}{\frac{\pi^{\frac{N-1}{2}}}{\left(\frac{N-1}{2}\right) !} \mathcal{R}^{N-1}}=\frac{r_{i}^{N-1}}{\mathcal{R}^{N-1}}
$$

Therefore:

$$
f_{\mathbf{r}_{i}}\left(r_{i}\right)=\frac{d F_{\mathbf{r}_{i}}\left(r_{i}\right)}{d r_{i}}=\frac{(N-1) r_{i}^{N-2}}{\mathcal{R}^{N-1}} \quad 0 \leq r_{i} \leq \mathcal{R}
$$

Now in order to solve equation (6), the probability distribution function of a new random variable corresponding to $\mathbf{r}_{1} \mathbf{r}_{2} \ldots \mathbf{r}_{k}$ must be found. First, we define a new random variable $\mathbf{r}_{i}^{\prime}$ as:

$$
\mathbf{r}_{i}^{\prime} \triangleq \frac{\mathbf{r}_{i}}{\mathcal{R}}
$$

Therefore, the PDF of $\mathbf{r}_{i}^{\prime}$ can be computed as [6]:

$$
f_{\mathbf{r}_{i}^{\prime}}\left(r_{i}^{\prime}\right)=(N-1) r_{i}^{\prime N-2} \quad 0 \leq r_{i}^{\prime} \leq 1
$$

From equations (6) and (12), the distortion of $k$-stage MP is:

$$
D_{\mathrm{MP}}=\|f\|^{2} \mathcal{R}^{2 k}\left(r_{1}^{\prime} r_{2}^{\prime} \ldots r_{k}^{\prime}\right)^{2}
$$

Thus, in order to find $D_{\mathrm{MP}}$, the PDF of $\mathbf{r}_{1}^{\prime} \mathbf{r}_{2}^{\prime} \ldots \mathbf{r}_{k}^{\prime}$ must be calculated. We use lemma 1 to find this probability distribution.

Lemma 1 Suppose $\mathbf{x}$ and $\mathbf{y}$ are independent random variables with PDF's:

$$
f_{\mathbf{y}}(y)=\alpha y^{\beta} \quad 0 \leq y \leq 1, f_{\mathbf{x}}(x)=a x^{\beta}(\ln x)^{\rho-1} \quad 0 \leq x \leq 1
$$$$
\text { Let } \mathbf{z}=\mathbf{x y} \text {. Then: }
$$

$$
f_{\mathbf{z}}(z)=-\frac{\alpha a}{\rho} z^{\beta}(\ln z)^{\rho} \quad 0 \leq z \leq 1
$$

where $\alpha, \beta$, a and $\rho$ are constants.

Proof: Let $\mathbf{w}$ be an auxiliary random variable defined by $\mathbf{w}=$ $\mathbf{x}$. The determinant of the Jacobian matrix is $J(x, y)=-w$. Therefore the PDF of $\mathbf{z}$ can be written as [6]:

$$
f_{\mathbf{z}}(z)=\int f_{\mathbf{z w}}(z, w) d w=\int \frac{1}{|w|} f_{\mathbf{x y}}\left(w, \frac{z}{w}\right) d w
$$

For nonzero probability distribution functions, $x$ and $y$ must be between 0 and 1 . Therefore, $0 \leq w \leq 1$ and $0 \leq \frac{z}{w} \leq 1$. This yields the boundaries of the integral equation as $z \leq w \leq 1$. The independence of the two random variables implies that:

$$
f_{\mathbf{z}}(z)=\int_{z}^{1} \frac{1}{w} f_{\mathbf{x}}(w) f_{\mathbf{y}}\left(\frac{z}{w}\right) d w
$$

Substituting the PDF's of $\mathbf{x}$ and $\mathbf{y}$ from (15) into (18):

$$
f_{\mathbf{z}}(z)=\int_{z}^{1} \frac{a}{w} w^{\beta}(\ln w)^{\rho-1} \alpha \frac{z^{\beta}}{w^{\beta}} d w=a \alpha z^{\beta} \int_{z}^{1}(\ln w)^{\rho-1} \frac{d w}{w}
$$

Therefore, the solution of the integral equation is:

$$
f_{\mathbf{z}}(z)=-\frac{\alpha a}{\rho} z^{\beta}(\ln z)^{\rho} \quad 0 \leq z \leq 1
$$


From equation (13) the PDF's of $\mathbf{r}_{1}^{\prime}$ and $\mathbf{r}_{2}^{\prime}$ have the same form as equation (15) with $\alpha=a=N-1, \beta=N-2$ and $\rho=1$ and $\mathbf{r}_{1}^{\prime}$ and $\mathbf{r}_{2}^{\prime}$ are independent. Therefore, using Lemma 1, the PDF of $\mathbf{z}_{2} \triangleq \mathbf{r}_{1}^{\prime} \mathbf{r}_{2}^{\prime}$ can be written as:

$$
f_{\mathbf{z}_{2}}\left(z_{2}\right)=-(N-1)^{2} z_{2}^{N-2}\left(\ln z_{2}\right) \quad 0 \leq z_{2} \leq 1
$$

Equation (21) has the same form as the PDF of $x$ in Lemma 1 with $a=-(N-1)^{2}, \beta=N-2$ and $\rho=2$. The PDF of $\mathbf{r}_{3}^{\prime}$ (equation (13)) has also the same form as the PDF of $y$ in Lemma 1 with $\alpha=N-1$ and $\beta=N-2$ and $\mathbf{r}_{3}^{\prime}$ and $\mathbf{z}_{2}=\mathbf{r}_{1}^{\prime} \mathbf{r}_{2}^{\prime}$ are independent. Thus, the PDF of $\mathbf{z}_{3} \triangleq \mathbf{z}_{2} \mathbf{r}_{3}^{\prime}=\mathbf{r}_{1}^{\prime} \mathbf{r}_{2}^{\prime} \mathbf{r}_{3}^{\prime}$ can be found using Lemma 1:

$$
f_{\mathbf{z}_{3}}\left(z_{3}\right)=\frac{(N-1)^{3}}{2} z_{3}^{N-2}\left(\ln z_{3}\right)^{2} \quad 0 \leq z_{3} \leq 1
$$

Continuing this for $k$ times, the PDF of $\mathbf{z}_{k} \triangleq \mathbf{r}_{1}^{\prime} \mathbf{r}_{2}^{\prime} \ldots \mathbf{r}_{k}^{\prime}$ will be:

$$
f_{\mathbf{z}_{k}}\left(z_{k}\right)=\frac{(N-1)^{k}(-1)^{k-1}}{(k-1) !} z_{k}^{N-2}\left(\ln z_{k}\right)^{k-1} \quad 0 \leq z_{k} \leq 1
$$

Now $E\left(z_{k}^{n}\right)=\int_{0}^{1} z_{k}^{n} f_{\mathbf{z}_{k}}\left(z_{k}\right) d z_{k}$ can be computed by:

$$
E\left(\mathbf{z}_{k}^{n}\right)=\int_{0}^{1} z_{k}^{n} \frac{(N-1)^{k}(-1)^{k-1}}{(k-1) !} z_{k}^{N-2}\left(\ln z_{k}\right)^{k-1} d z_{k}
$$

Let $y=-(n+N-1) \ln z_{k}$. Therefore, $z_{k}=e^{-\frac{y}{n+N-1}}, d z_{k}=$ $-e^{-\frac{y}{n+N-1}} d y /(n+N-1)$ and $0 \leq y \leq \infty$. Thus:

$$
E\left(\mathbf{z}_{k}^{n}\right)=\frac{(N-1)^{k} \int_{0}^{\infty} y^{k-1} e^{-y} d y}{(k-1) !(n+N-1)^{k}}=\frac{(N-1)^{k} \Gamma(k)}{(k-1) !(n+N-1)^{k}}
$$

Since $\Gamma(k)=(k-1)$ ! for integer $k$, the expectation of $\mathbf{z}_{k}^{n}$ is:

$$
E\left(\mathbf{z}_{k}^{n}\right)=\left(\frac{N-1}{n+N-1}\right)^{k}
$$

Substituting (26) into (14), the average distortion of $k$-stage matching pursuit for uniform signals and dictionaries can be found by:

$$
D_{\mathrm{MP}}=\|f\|^{2} \mathcal{R}^{2 k}\left(\frac{N-1}{N+1}\right)^{k}
$$

\section{OPTIMIZATION}

The total distortion of MP encoder is the distortion caused by the remaining residual signal calculated by equation (27) plus the distortion caused by quantizing the inner product coefficients at each stage. Suppose the inner product coefficients are quantized by a uniform quantizer with $q$ quantization steps. The distribution of inner product coefficients is rather complex. In this paper we assume the coefficients are uniformly distributed in the range of the quantizer. Our simulation results for random signals show that this assumption does not cause a significant error in our calculations. With this assumption, the quantization distortion can be written as:

$$
D_{\mathrm{Q}}=\sum_{j=0}^{k-1} \frac{\|f\|^{2}}{12 q^{2}}=\frac{k\|f\|^{2}}{12 q^{2}}
$$

The rate of a $k$-stage MP encoder can be found by:

$$
\text { Rate }=k \log _{2} q+k \log _{2} M
$$

Now the problem is to find the optimum number of MP stages $k$ and quantization levels $q$ to minimize the distortion for a given signal dimension, dictionary size and rate budget. The Lagrangian multiplier method is used to solve this optimization problem:

$$
J(\lambda)=D_{\mathrm{MP}}+D_{\mathrm{Q}}+\lambda \text { Rate }
$$

where $\lambda$ is the Lagrangian multiplier. Substituting (27), (28) and (29) into (30), the Lagrangian cost function can be formulated as:

$J(\lambda)=\|f\|^{2} \mathcal{R}^{2 k}\left(\frac{N-1}{N+1}\right)^{k}+\frac{k\|f\|^{2}}{12 q^{2}}+\lambda\left(k \log _{2} q+k \log _{2} M\right)$

It is easy to show that:

$$
\frac{\partial J(\lambda)}{\partial q}=0 \Rightarrow q_{\mathrm{opt}}=\sqrt{\frac{\|f\|^{2} \ln 2}{6 \lambda}}
$$

Substituting equation (32) into equation (28) yields:

$$
D_{\mathrm{Q}}=\frac{k \lambda}{2 \ln 2}
$$

The optimal number of MP stages $(k)$ can be found by solving:

$$
\begin{gathered}
\frac{\partial J(\lambda)}{\partial k}=\|f\|^{2} \mathcal{R}^{2 k}\left(\frac{N-1}{N+1}\right)^{k} \ln \left(\mathcal{R}^{2}\left(\frac{N-1}{N+1}\right)\right) \\
+\frac{\lambda}{2 \ln 2}+\lambda \log _{2} \sqrt{\frac{\|f\|^{2} \ln 2}{6 \lambda}}+\lambda \log _{2} M=0
\end{gathered}
$$

Thus:

$$
\frac{\partial J(\lambda)}{\partial k}=0 \Rightarrow k_{\mathrm{opt}}=\frac{\ln \left(\frac{\frac{\lambda}{2 \ln 2}+\lambda \log _{2} \sqrt{\frac{\|f\|^{2} \ln 2}{6 \lambda}}+\lambda \log _{2} M}{-\|f\|^{2} \ln \left(\mathcal{R}^{2}\left(\frac{N-1}{N+1}\right)\right)}\right)}{\ln \left(\mathcal{R}^{2}\left(\frac{N-1}{N+1}\right)\right)}
$$

Now that the optimum values for $k$ and $q$ have been found, the next step is to find the optimal $\lambda$ that satisfies the bit budget constraint and minimizes equation (31). Assuming the bit budget is fixed and is denoted by $R_{\text {budget }}$ :

$$
R_{\text {budget }}=k \log _{2} q+k \log _{2} M
$$

Since both $k$ and $q$ are functions of $\lambda$, equation (36) can be solved numerically to find $\lambda$ for any value of $M, N$ and $R_{\text {budget. }}$.

\section{SIMULATION RESULTS}

In this section, first the accuracy of our analytical result for the MP distortion is verified by comparison to practical matching pursuit encoders. Then, our optimization scheme is evaluated and at the end, the application of our optimization method for nonuniform signals and dictionaries is discussed.

Figure 2.(a) shows the rate distortion curve of the matching pursuit encoding of 8 dimensional random samples with a dictionary of 128 random elements. The inner product coefficients are quantized by a 3 bit quantizer and the rate distortion curve is compared by what is predicted by equations (27) and (28) $(D=$ $\left.D_{M P}+D_{Q}\right)$. As can be seen in the figure, our prediction is very close to the experimental results although a random dictionary is used instead of the optimal dictionary with elements uniformly distributed on the unit $N$-sphere. Figure 2.(b) compares the experi- 

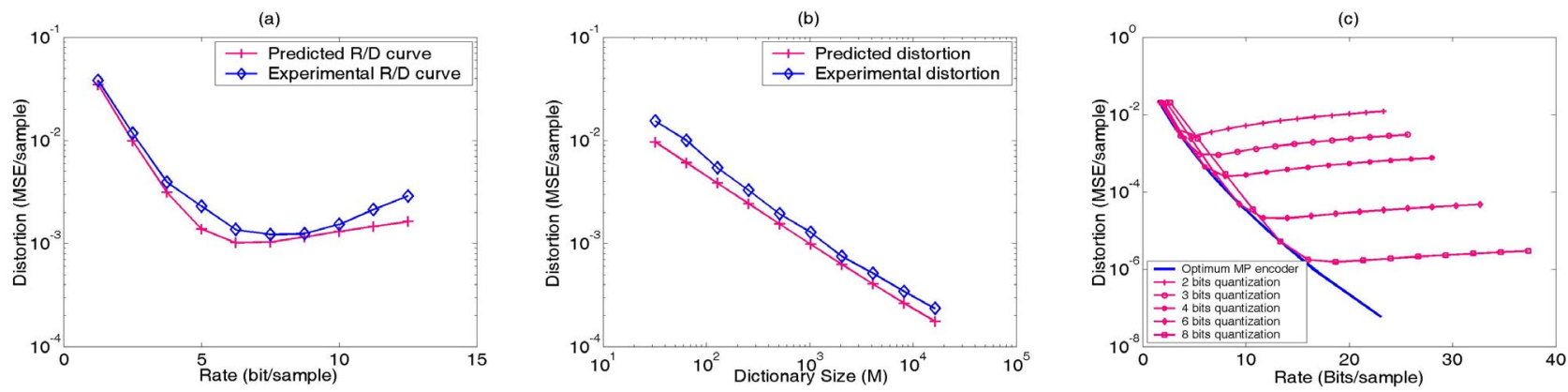

Fig. 2. (a) Comparison of rate distortion curves for $N=8, M=128$ and $q=8$. (b) Comparison of distoritons predicted by our equations and experimental data for $N=16, k=5$ and $q=32$. (c) Rate distortion curves for optimum MP encoder and other MP encoders

mental and predicted distortions for a 5-stage matching pursuit encoder using a 5 bit quantizer. The input is 16-dimensional random signal and the dictionary elements are random. The distortion is obtained for different dictionary sizes and as shown in figure 2.(b) the experimental distortion is very close to our prediction which proves the accuracy of our calculations.

Now that the accuracy of our model is verified, the optimized MP encoder must be evaluated. Figure 2.(c) shows the predicted distortions for several MP encoders along with the rate distortion curve of the optimized MP encoder. As shown in the figure, the optimized encoder selects the optimal number of MP stages and quantization step numbers to provide the best performance among all encoders for different bit rates.

\begin{tabular}{|c|c|c|c|c|c|c|c|}
\hline Rate & $k$ & $q$ & PSNR & & & & \\
\hline $0.37 \mathrm{bpp}$ & $2 *$ & $4 *$ & $25.06 \mathrm{~dB}$ & Rate & $k$ & $q$ & PSNR \\
\hline $0.37 \mathrm{bpp}$ & 1 & 16384 & $24.14 \mathrm{~dB}$ & $1.01 \mathrm{bpp}$ & $5^{*}$ & $8^{*}$ & $24.96 \mathrm{~dB}$ \\
\hline $1.01 \mathrm{bpp}$ & 5* & 8* & $27.17 \mathrm{~dB}$ & $1.00 \mathrm{bpp}$ & 4 & 64 & $24.81 \mathrm{~dB}$ \\
\hline $1.00 \mathrm{bpp}$ & 4 & 64 & $26.47 \mathrm{~dB}$ & $1.03 \mathrm{bpp}$ & 6 & 2 & $24.17 \mathrm{~dB}$ \\
\hline $1.03 \mathrm{bpp}$ & 6 & 2 & $26.91 \mathrm{~dB}$ & $0.81 \mathrm{bpp}$ & $4 *$ & $8 *$ & $24.61 \mathrm{~dB}$ \\
\hline $1.42 \mathrm{bpp}$ & 7* & $8^{*}$ & $27.93 \mathrm{~dB}$ & $0.84 \mathrm{bpp}$ & 3 & 256 & $24.34 \mathrm{~dB}$ \\
\hline $1.50 \mathrm{bpp}$ & 6 & 64 & $27.80 \mathrm{~dB}$ & $0.86 \mathrm{bpp}$ & 5 & 2 & $24.03 \mathrm{~dB}$ \\
\hline $1.50 \mathrm{bpp}$ & 8 & 4 & $27.77 \mathrm{~dB}$ & $1.97 \mathrm{bpp}$ & 9* & $16^{*}$ & $25.78 \mathrm{~dB}$ \\
\hline $1.97 \mathrm{bpp}$ & 9* & $16^{*}$ & $28.61 \mathrm{~dB}$ & $2.00 \mathrm{bpp}$ & 8 & 64 & $25.78 \mathrm{~dB}$ \\
\hline $2.00 \mathrm{bpp}$ & 8 & 64 & $28.47 \mathrm{~dB}$ & $2.03 \mathrm{bpp}$ & 10 & 8 & $25.71 \mathrm{~dB}$ \\
\hline $2.03 \mathrm{bpp}$ & 10 & 8 & $28.60 \mathrm{~dB}$ & $1.97 \mathrm{bpp}$ & 7 & 256 & $25.68 \mathrm{~dB}$ \\
\hline $1.97 \mathrm{bpp}$ & 7 & 256 & $28.21 \mathrm{~dB}$ & & & & \\
\hline
\end{tabular}

Table 1. Left table: results for Hill, Right table: results for Lenna.

Although we have only considered uniformly distributed signals and dictionaries during derivation of our equations, our simulation results show that our optimized MP encoder exhibits optimum performance for other signal distributions and dictionaries. We have compared our optimal MP encoder with MP encoders with different number of MP stages and different quantizers for two images ( $256 \times 256$ Lenna and Hill images). The dictionary is the Gabor dictionary as suggested in [4] with 1024 elements and the block size is $8 \times 8$. Table 1 compares the PSNR's of different MP encoders for different bit rates. The optimum encoder according to our optimization in section 4 is indicated in the table by a star for each bit rate. As shown in the tables the PSNR obtained by the optimum MP encoder is the highest among other possible encoders in all cases. This shows that although our optimized MP encoder is derived for uniform signals and dictionaries, it can be used to op- timize MP encoders for other signal distributions. One reason for this is that uniform signals have the highest entropy among other distributions for discrete signals and consequently are among the most difficult ones to compress. Therefore, the MP distortion for uniform signals is generally higher than the distortion for other signal distributions. In fact, equation (27) presents an upper bound for the MP distortion of nonuniform signals. In other words, the average distortion for the worst signal distribution is the upper bound for the average distortion of arbitrary signal distributions. Since this upper bound is not very conservatively selected (in contrast to the one used in [2] which considers the worst case scenario at every stage of matching pursuit for arbitrary dictionaries), it can be used to efficiently optimize the MP system for other distributions. Another advantage of our optimization method is that it does not require any adaptation and can be mathematically computed as opposed to methods used in $([2,3])$.

\section{CONCLUSION}

In this paper, matching pursuit distortion is analytically expressed in terms of MP encoder parameters for uniform signals and dictionaries. This analytically derived distortion was used to find the optimal MP encoder. Our simulation results showed that not only the analytical derivation is very accurate, but also the optimum MP encoder for uniformly distributed signals exhibits optimum performance for other signal distributions and dictionaries.

\section{REFERENCES}

[1] S.G. Mallat, Z. Zhang, "Matching pursuits with time-frequency dictionaries" IEEE Tran. SP, vol. 41, pp. 3397-3415,1993.

[2] P. Frossard, P. Vandergheynst, R.M. Figueras i Ventura, M. Kunt, "A posteriori quantization of progressive matching pursuit streams" IEEE Tran. SP, vol. 52, pp. 525-535, 2004.

[3] Q. Liu, Q. Wang, L. Wu, "Size of the dictionary in matching pursuit algorithm," IEEE Tran. SP, vol. 52, pp. 3403-3408, 2004.

[4] R. Neff, A. Zakhor, "Modulus quantization for matching-pursuit video coding," IEEE Tran. CSVT, vol. 10, pp. 895-912, 2000.

[5] K. T. McDonald, "Volume and surface area of an N-sphere," http://www.hep.princeton.edu/ mcdonald/examples/nsphere.pdf.

[6] A. Papoulis, "Probability, Random Variables, and Stochastic Processes", McGraw-Hill, $2^{\text {nd }}$ ed., 1984. 